\title{
PRODUÇÃO DE MATERIAL DIGITAL PARA O ENSINO DE ENFERMAGEM
}

\author{
Ana Luísa Cogo - UFRGS \\ Gabriela Perry - Universidade Federal do Rio Grande do Sul \\ Marlise B Santos - UFRGS
}

\section{RESUMO:}

As tecnologias educacionais digitais colaboram com a simulação de situações práticas no ensino de Enfermagem, como estratégia que procura garantir a segurança do paciente possibilitando a antecipação da realização destes procedimentos em laboratório de ensino ou como fonte de consulta em casos de dúvidas. Este relato tem como objetivo descrever as características e a metodologia de desenvolvimento do material digital, no formato de vídeo, intitulado punção venosa periférica com cateter. A produção do mesmo contou com uma equipe multiprofissional. As etapas observadas na produção do material digital foram definição dos objetivos instrucionais e o público alvo; a determinação do formato, do orçamento e do cronograma; a seleção do conteúdo; o desenvolvimento das estratégias para avaliar o vídeo e a sua efetividade educacional; o desenvolvimento do roteiro ou storyboard; o desenvolvimento do protótipo e a avaliação do produto e do processo. A produção de vídeos educacionais constitui-se em importante material digital de apoio á aprendizagem e a possibilidade de integrar uma equipe de especialistas na sua elaboração qualifica o processo.

PALAVRAS-CHAVES: Mídia Audiovisual. Tecnologia Educacional. Educação a Distância. Educação Superior.

\section{DESIGNING A DIGITAL RESOURCE FOR NURSING EDUCATION}

\section{ABSTRACT:}

Educational technologies cooperate to the simulation of practical situations in nursing education as a strategy that seeks to guarantee the patient's safety by anticipating the procedures in educational labs or as a resource in case of doubts. This narrative seeks to describe the features and methodology used to develop a digital resource, in a video format, named puncture by peripheral venous catheter. A multidisciplinary team was needed in order to design the resource. The stages observed in the production of the video were: the definition of the audience and the instructional objectives; the delimitation of the format, the budget and schedule; the selection of content, the development of evaluation strategies and its educational effectiveness; the development of a script or storyboard, the development of a model and the evaluation of the process and final product. Educational videos are important learning support resources and the possibility of having a multidisciplinary team qualifies the whole design process.

KEYWORDS: Video-Audio Media. Education Technology. Education, Distance. Education, Higher.

\section{INTRODUÇÃO}

As tecnologias educacionais digitais tem sido desenvolvidas observando as características das áreas do conhecimento a que se referem. No caso da Enfermagem, a expansão da utilização dessas tecnologias no Brasil ocorreu após a publicação das Diretrizes Curriculares do Curso de Enfermagem (BRASIL, 2001). Esta legislação incentivou a formação do profissional enfermeiro preparado para utilizar diversos recursos tecnológicos nas suas práticas assistenciais e educativas.

Entre as características do curso de graduação em Enfermagem pode-se destacar a necessidade de associar a teoria com a prática no intuito de qualificar um profissional 
crítico-reflexivo (BRASIL, 2001). O propósito educativo não pode se restringir à memorização de um conteúdo ou ao desenvolvimento isolado de uma habilidade prática. Neste contexto o ensino de fundamentos de enfermagem, área que se refere às habilidades clínicas e procedimentais, conta com o apoio das tecnologias educacionais, pois serão apresentadas na fase inicial do curso sendo aperfeiçoadas como tema transversal ao longo de toda a graduação (COGO et al., 2007). A possibilidade de apresentar materiais problematizadores das práticas clínicas que simulem a tomada de decisão irá colaborar com a aprendizagem, sendo um recurso em atenção a manutenção da segurança dos pacientes durante a execução de procedimentos (KILLAM et al., 2010).

A punção venosa periférica com cateter é um procedimento invasivo que consiste na instalação de um dispositivo plástico sobre agulha em uma veia periférica com utilização de técnica asséptica (LYNN, 2012). Devido à necessidade de empregar conhecimentos de anatomia, fisiologia e farmacologia, entre outros, bem como do risco de infecção que pode ser originado deste procedimento, é que o profissional habilitado a realizá-lo é o enfermeiro. Em estudo que avaliou a adequação da técnica de punção venosa realizada por enfermeiros foi identificado que havia a necessidade de aprimorarem as habilidades técnico-científicas (TORRES; ANDRADE; SANTOS, 2005). Devido à complexidade na sua execução e por este ser um procedimento muito frequente em áreas hospitalares e ambulatoriais, é que se percebe a expectativa e a ansiedade do estudante de Enfermagem em desenvolver esta habilidade.

No intuito de apoiar o desenvolvimento de habilidades de Enfermagem materiais digitais tem sido desenvolvidos para apoiar as práticas de ensino presenciais ou como suporte a atividades a distância (FROTA et al., 2013). Vídeos, hipertextos e jogos educativos sobre punção venosa periférica foram produzidos para apoiar o ensino de Enfermagem estimulando a autonomia do estudante (DUCAN; YARWOOD-ROSS; HAIGH, 2013) e pretendendo minimizar a ansiedade frente a este procedimento ainda não realizado (LIMA et al., 2011; SILVA; COGO, 2007).

Desta forma, as tecnologias educacionais digitais colaboram com a simulação de situações práticas no ensino de Enfermagem, como uma estratégia que procura garantir a segurança do paciente através da antecipação a realização destes procedimentos em laboratório de ensino ou como fonte de consulta em casos de dúvidas. Além disso, esses materiais educativos possuem a qualidade de interoperabilidade, podendo ser distribuídos para uma grande quantidade de estudantes e profissionais, além de serem acessados várias vezes, respeitando os diferentes ritmos de aprendizagem (COGO et al., 2007).

Estudos que avaliaram a usabilidade de materiais digitais (hipertexto, jogo educativo e simulação) sobre procedimentos de Enfermagem identificaram que os estudantes de Enfermagem os consideraram adequados quanto ao conteúdo e a finalidade, mas tiveram dificuldade na visualização e no salvamento do material (SCHATKOSKI et al., 2007). Acredita-se que com a disseminação e popularização das tecnologias da informação e da comunicação, como também a ampliação ao acesso às tecnologias móveis, faça com que os estudantes de ensino superior estejam mais fluentes na utilização destes recursos. E esta evolução pode ser percebida nas propostas de desenvolvimento de OA para equipamentos de telefonia móvel. Como exemplo citase o desenvolvimento de um aplicativo sobre 'mensuração da pressão venosa central' a ser acessado em smartphone por estudantes de Enfermagem em práticas hospitalares (GALVÃO; PÜSCHEL, 2012). 
Entre os formatos de materiais digitais encontram-se os vídeos. De la Serna (1994) define vídeo educacional como todo vídeo que foi projetado, produzido e avaliado para ser inserido em um processo concreto de aprendizagem de forma criativa e dinâmica. Esta definição associa a qualificação do vídeo como educacional a partir das intenções dos produtores, e não de seus efeitos sobre a aprendizagem. Além disso, a definição não abarca de forma clara situações não formais e não planejadas de aprendizagem. Salinas (1992) e Graells (2010) apresentam uma definição mais aberta, e que nos parece mais apropriada, que caracteriza um vídeo como educacional quando ele está inserido em uma atividade de ensino. Graells (2010) propôs uma tipologia para vídeos educacionais, classificando-os como: documentais, narrativos, lição monoconceitual, lição temática e vídeo motivador.

Os vídeos educacionais em saúde podem destacar vários aspectos visando a apresentação de um caso clínico, mas quando há a necessidade de aprofundar a discussão de forma crítica pode haver a perda do foco, conforme identificado em um estudo com estudantes de Enfermagem (ROY; MCMAHOM, 2012). Em estudo que comparou dois grupos de estudantes de Enfermagem em aulas sobre administração de medicamentos foi evidenciado que o grupo que teve acesso ilimitado a um vídeo sobre o tema teve um desempenho clínico melhor e maior satisfação na avaliação desta atividade de ensino (HOLLAND et al., 2012). Assim, destaca-se a importância de haver um planejamento detalhado para o desenvolvimento do vídeo e sua avaliação durante esta fase.

Provavelmente uma das diretrizes de orientação sobre design instrucional para materiais digitais mais conhecida seja a de Merrill (2002), que revisou diversos autores da área e identificou cinco princípios comuns em todos eles, os quais são: os estudantes devem estar engajados em resolver problemas reais; conhecimentos existentes devem ser utilizados como fundação para novos conhecimentos; o conhecimento deve ser demonstrado ao aprendiz; o conhecimento deve ser aplicado pelo aprendiz; o conhecimento deve ser integrado ao contexto do estudante. Gardner (2010) reconhece que apesar da aplicação destes princípios estarem associados com ganhos na aprendizagem dos estudantes, eles são difíceis de serem aplicados, pois requerem muito tempo de planejamento. Por este motivo, este autor oferece um roteiro de perguntas que pode ser utilizado para alavancar a aplicação destes princípios de forma mais rápida.

Amparados nestes relatos de sucesso na adoção de práticas de desenvolvimento de material digital, e reconhecendo no vídeo uma mídia adequada aos objetivos de aprendizagem da disciplina Cuidados de Enfermagem ao Adulto I (ENF01015), relatamos, neste artigo, o processo de produção do vídeo "Punção Venosa Periférica com Cateter". Este relato tem como objetivo descrever as características e a metodologia de desenvolvimento do material digital, no formato de vídeo, intitulado punção venosa periférica com cateter.

\section{DESENVOLVIMENTO DO VÍDEO}

O vídeo denominado "Punção Venosa Periférica com Cateter" (http://videos.ufrgs.br/sead/napead/puncao-venosa-periferica-com-cateter) foi produzido pela Escola de Enfermagem e o Núcleo de Apoio Pedagógico a Distância (NAPEAD) da Universidade Federal do Rio Grande do Sul (UFRGS). A equipe proponente buscou o apoio do NAPEAD para a realização do vídeo, pois buscavam o apoio técnico e pedagógico para a realização deste projeto que foi contemplado em Edital interno da 
Universidade com apoio para esta finalidade. O Núcleo possui além de pessoal técnico, equipamentos profissionais e um estúdio para a produção de vídeos.

O vídeo foi proposto por uma professora da disciplina que desenvolve temas de fundamentos de Enfermagem e duas enfermeiras que atuam no laboratório de práticas da Instituição, com o apoio de estudantes do curso de graduação em Enfermagem. Um dos estudantes de Enfermagem colaborou na busca bibliográfica de etapas do procedimento para elaboração do roteiro, o outro foi o ator demonstrando a realização do procedimento.

A produção do mesmo contou com a equipe interdisciplinar do Núcleo de Apoio Pedagógico à Educação a Distância (NAPEAD), representada por um estudante de Design Gráfico, uma bacharel em Artes Visuais e uma bacharel em Pedagogia. O estudante de Design ficou responsável pelas ilustrações e animações; a artista plástica realizou a filmagem e a edição, e a pedagoga orientou os trabalhos e mediou a comunicação com a equipe da Escola de Enfermagem.

Existem diferentes diretrizes que orientam a produção de material digital. Neste projeto as proponentes observaram as etapas propostas para o desenvolvimento de recursos multimídia de Frey e Sutton (2010), os quais foram: definir os objetivos instrucionais e o público alvo; rever e investigar em repositórios outras opções existentes; determinar o formato, o orçamento e o cronograma; determinar o conteúdo, as atividades e estratégias de avaliação; desenvolver estratégias para avaliar a efetividade educacional; desenvolver o roteiro ou storyboard; desenvolver um protótipo; realizar uma avaliação performativa; completar o projeto e realizar uma avaliação do produto e do processo.

Na etapa 1 a equipe da Escola de Enfermagem realizou a definição dos objetivos de ensino e de aprendizagem a serem alcançados. Sendo assim, pretendeu-se apresentar de forma objetiva os materiais utilizados no procedimento punção venosa com cateter e as etapas para sua realização, aproximando-se da realidade vivenciada no hospital que os estudantes da quarta a nona etapa do curso de graduação em Enfermagem da UFRGS encontrarão suas práticas curriculares.

Na etapa 2 houve a revisão e a análise de outros materiais disponíveis sobre o tema, o que reforçou a importância de elaborar-se um vídeo próprio, pois muitos destes continham conteúdos inadequados, não observação de princípios éticos ao expor usuários ou usando modelos vivos, como também foram localizados materiais em outros idiomas. Na etapa 3 foi determinado o formato e o planejamento dos recursos, o qual deveria ser compatível com aparelhos de telefonia móvel, de curta duração e utilizando os recursos disponíveis na Universidade. Foram utilizados materiais do Laboratório de Práticas da Escola de Enfermagem (cateteres, algodão, extensores, seringas, caixa de descarte de perfuro-cortante, o braço simulador de punções venosas). Em observação com os princípios de segurança, optou-se por realizar o procedimento em uma peça de simulação de baixa fidelidade e o executor do procedimento foi um estudante de Enfermagem com destreza na realização do mesmo, que atuou como voluntário. O NAPEAD já contava com a infra-estrutura de equipamentos (câmeras, cabos e microfones) para a gravação e o software de edição, e os recursos humanos para desenvolverem o material.

Na etapa 4 houve a determinação do conteúdo, das atividades e das estratégias de avaliação, como foi relatado anteriormente, o vídeo foi planejado observando a sistemática de execução do procedimento de punção venosa baseado em evidências e segundo o preconizado nas Instituições nas quais são realizadas pelos estudantes.

Na etapa 5 houve a determinação dos critérios e dos instrumentos de avaliação da efetividade do projeto, estabelecendo-se que o conteúdo seria avaliado por professores 
da área de fundamentos de Enfermagem e a usabilidade com os estudantes da quarta etapa do curso, em formulário previamente utilizado na avaliação de materiais digitais previamente desenvolvidos pela professora responsável pelo planejamento.

$\mathrm{Na}$ etapa 6 foi desenvolvido o roteiro com a estrutura em três colunas contendo o áudio (música, som, locutor), vídeo (imagem, título), letreiros. Este roteiro foi submetido ao Edital e quando o projeto foi aprovado o mesmo sofreu adaptações após ser avaliado pela equipe do NAPEAD. Nesta fase é que efetivamente iniciou a produção do vídeo.

A partir de reuniões e encontros das duas equipes o conteúdo do vídeo foi sendo apropriado pela equipe do NAPEAD. Foram, aos poucos, se estabelecendo alguns direcionamentos sobre como o vídeo seria desenvolvido. Salienta-se que nesta fase do projeto a conversa e o diálogo entre todos os envolvidos foi importante não só para o entendimento do que se busca fazer, como para o estabelecimento de vínculos entre os participantes. Na vivência do NAPEAD, há a constatação de que a pouca participação das equipes proponentes, o que não foi o caso neste projeto, acarreta dúvidas, atrasos e pouca compreensão da parte da equipe técnica. Em pesquisa anterior realizada, que analisou a metodologia de implementação de objetos de aprendizagem neste Núcleo, apontou para a necessidade de entrevistas e reuniões pedagógicas e técnicas para a explicitação do projeto e compreensão por toda a equipe envolvida (SILVEIRA; CARNEIRO, 2012).

Após a etapa inicial de reuniões entre todos os participantes foi agendada a gravação do vídeo. Por questões de segurança, o vídeo foi realizado em estúdio e em uma peça de simulação de baixa fidelidade. Quem realizou o procedimento foi um estudante do curso de Enfermagem que já dominava a técnica e atuou como ator voluntário (FIGURA 1).

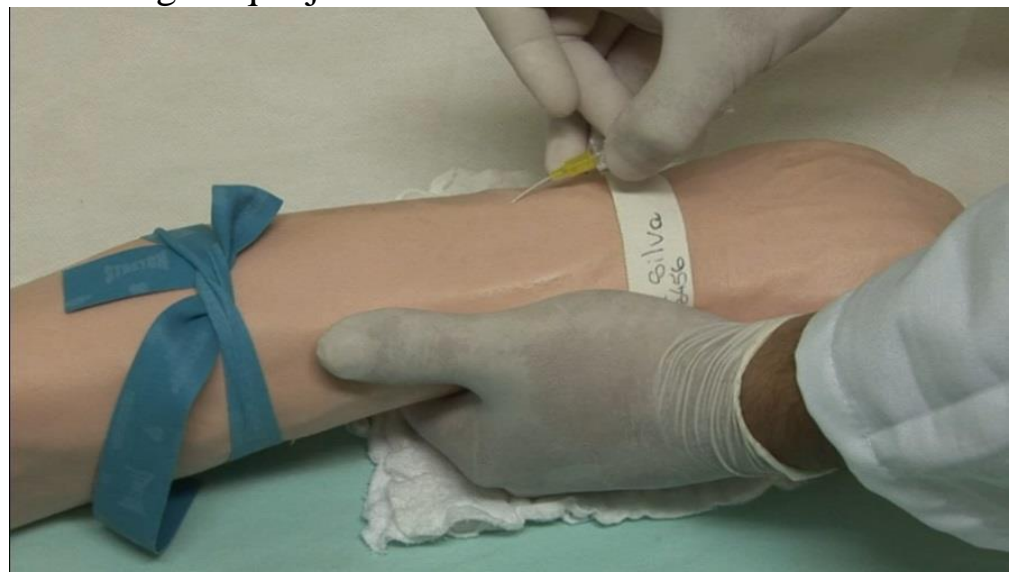

FIGURA 1. Printscreen da tela com a execução do procedimento em simulador de baixa fidelidade.

A seguir foi realizada a etapa de animações do vídeo, pois havia no projeto alguns momentos durante o vídeo em que se demonstrava o braço visto por dentro, com suas veias e artérias, apontando a trajetória da agulha ao realizar a punção venosa (FIGURA 2). Houve nesta etapa a necessidade da busca de referências mais realistas, o que foi um desafio maior para o ilustrador e designer. A professora coordenadora do projeto percebeu a complexidade da tarefa e reuniu um grande número de referências para apoiar a compreensão do que deveria ser realizado. 


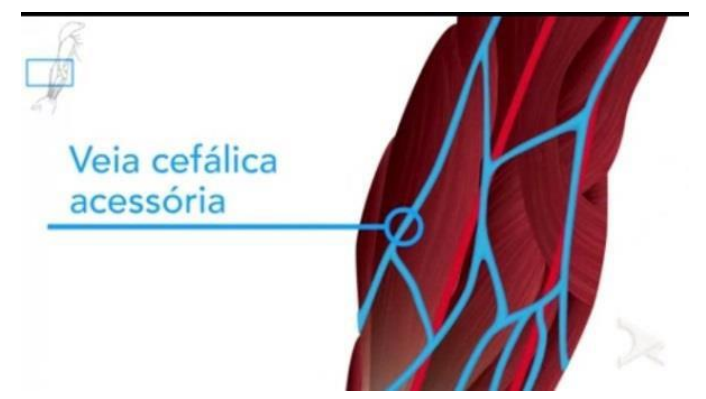

FIGURA 2- Printscreen do desenho com referências anatômicas.

O roteiro também previa a inserção de fotografias de cateteres, e para tal foi realizada uma sessão de fotos na qual a presença da professora responsável, apontando e direcionando o trabalho foi essencial (FIGURA 3). O enquadramento das cenas e das animações foi projetado especialmente para aparelhos de telefonia móvel, o que permite que os estudantes os acessem durante os diferentes cenários de suas práticas curriculares, oferecendo informações que contribuem para a realização dos procedimentos. A integração do vídeo com recursos de animação e imagens pretende contextualizar a técnica da punção venosa, oferecendo maiores detalhes que estão envolvidos na sua execução.

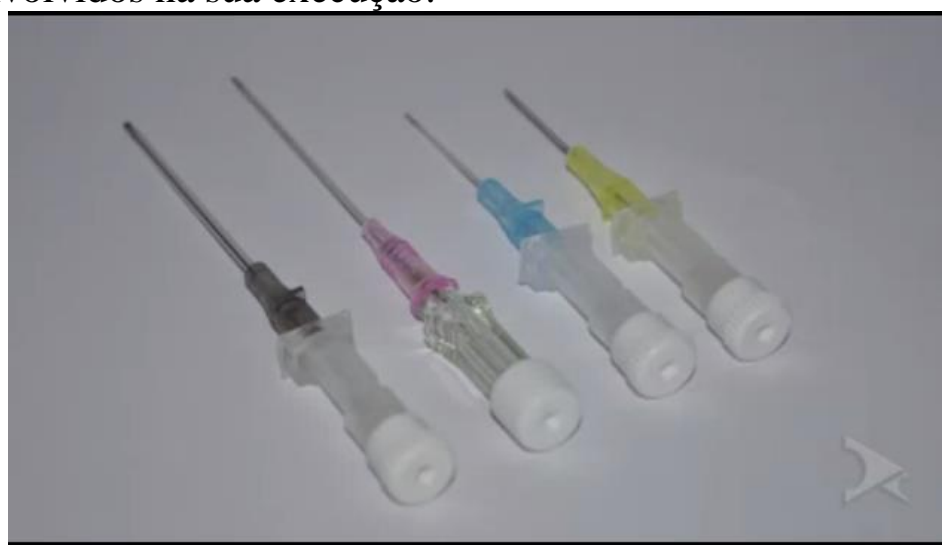

FIGURA 3-Printscreen da tela com as fotos dos materiais.

Na etapa 7 foi desenvolvido o protótipo disponibilizado à equipe para avaliação e foram realizadas adequações. Na etapa 8 houve a avaliação inicial do material, quando este foi disponibilizado a três professores da área da Enfermagem com conhecimento sobre o tema e três estudantes de graduação, que fizeram recomendações sobre alguns aspectos do vídeo que foram aperfeiçoados em uma nova edição. Foi interessante observar que poucas recomendações forem feitas, e o mesmo obteve aprovação com muita rapidez.

Na etapa 9 houve a conclusão do design, no qual foram realizados os ajustes finais de editoração. Na etapa 10, que se encontra o desenvolvimento deste vídeo no presente momento, refere-se a realização de avaliação do produto e do processo. Está em fase de realização uma investigação da usabilidade do vídeo com estudantes de Enfermagem da instituição, os quais terão acesso ao mesmo e poderão avaliar com maior precisão a adequação deste recurso em práticas curriculares.

O vídeo encontra-se disponível para acesso livre e gratuito no repositório do NAPEAD com licença Creative Commons - Atribuição-Não ComercialCompartilhadaIgual (CC BY-NC-AS). 


\section{CONSIDERAÇÕES FINAIS}

A produção de vídeos educacionais constitui-se em importante ferramenta de apoio à formação dos Enfermeiros que necessitam executar procedimentos invasivos sem colocar em risco a segurança de pessoas durante o seu processo de aprendizagem. $\mathrm{O}$ desenvolvimento deste projeto pela equipe da Escola de Enfermagem e do NAPEAD da UFRGS sinaliza a importância das Instituições de ensino superior investirem em equipes multiprofissionais qualificadas na produção de material digital de qualidade.

As limitações que ocorreram no desenvolvimento deste projeto foram relacionados a necessidade de desenvolver uma sistemática de trabalho entre as equipes responsáveis pelo conteúdo e pela produção do material digital. Essas equipes não haviam trabalhado anteriormente e após reuniões sistemáticas foi possível estabelecer um trabalho colaborativo. A fase de avaliação da usabilidade pelos estudantes é uma etapa que deverá ser concluída para que se obtenha uma avaliação ampliada do alcance dos objetivos deste projeto de desenvolvimento.

\section{REFERÊNCIAS BIBLIOGRÁFICAS}

BRASIL. CONSELHO NACIONAL DE EDUCAÇÃO. CÂMARA DE EDUCAÇÃO SUPERIOR. Resolução n.3, de 07 de novembro de 2001. Institui diretrizes curriculares nacionais do Curso de graduação em enfermagem. Diário Oficial da União, Brasília, DF, 9 de Nov. 2001. Disponível em:

<http://portal.mec.gov.br/cne/arquivos/pdf/CES03.pdf>. Acesso em: 02 nov. 2015.

SILVEIRA, M.S.; CARNEIRO, M.L.F.. Diretrizes para a Avaliação da Usabilidade de Objetos de Aprendizagem. In: Anais do $23^{\circ}$ Simpósio Brasileiro de Informática na Educação (SBIE 2012), 23, 2012, Rio de Janeiro. Disponível em: < http://www.brie.org/pub/index.php/sbie/article/view/1713/1474> Acesso em: 02 nov 2015.

COGO, A.L.P. et al. Desenvolvimento e utilização de objetos educacionais digitais no ensino de enfermagem. Revista Latino-americana de Enfermagem, Ribeirão Preto, v. 15, n. 4, , jul-ago 2007. Disponível em:< www.eerp.usp.br/rlae> Acesso em: 02 nov 2015

DE LA SERNA, M. C. Los vídeos didácticos: Claves para su producción y evaluación, en revista. Pixel, n. 1, p.: 31-42, 1994.

DUCAN, I.; YARWOOD-ROSS, L.; HAIGH, C.. YouTube as a source of clinical skills education. Nurse Education Today, Philadelphia, v. 33, n. 12, p.1576-1580, dez. 2013. Disponível em:

<http://www.sciencedirect.com/science/article/pii/S0260691712004108>. Acesso em: 26 abr. 2014.

FREY, B.A.; SUTTON, J.M.. A model for developing multimidea learning projects. MERLOT Journal of Online Learning and Teaching, 2010; V. 6, N. 2, p.: 491-507.

FROTA, N. M. et al. Construção de uma tecnologia educacional para o ensino de enfermagem sobre punção venosa. Revista Gaúcha de Enfermagem, Porto Alegre, v. 34, n. 2, p.29-36, jun. 2013. Disponível em: <http://seer.ufrgs.br/index.php/RevistaGauchadeEnfermagem/article/view/33258/26035 >. Acesso em: 02 nov. 2015 
GALVÃO, E.C.F.; PÜSCHEL, V.A.A. Aplicativo multimídia em plataforma móvel para o ensino da mensuração da pressão venosa central. Revista da Escola de Enfermagem da USP, São Paulo, v. 46, n. esp., p.: 107-15, 2012.

GARDNER, J. Applying Merrill's First Principles of Instruction: Practical Methods Based on a Review of the Literature. Educational Technology, v. 50, n. 2, p.:20-25, 2010

GRAELLS, P. M.. Los vídeos educativos: tipología, funciones, orientaciones para su uso. Disponível em: $<\mathrm{http} / / / \mathrm{www}$.peremarques.net/videoori.htm>. Acesso em: 03 nov. 2015.

HOLLAND, A. et al. Online video in clinical skills education of oral medication administration for undergraduate student nurses: A mixed methods, prospective cohort study. Nurse Education Today, V. 33, p.: 663-670, 2013.

KILLAM, L. et al. Views on Unsafe Nursing Students in Clinical Learning. International Journal of Nursing Education Scholarship, v. 7, n. 1, art. 36, 2010.

LIMA, I. C. V. et al. Comunicação entre acadêmicos de enfermagem e clientes com AIDS. Revista da Escola de Enfermagem da USP, São Paulo, v. 2, n. 45, p.426-432, abr. 2011. Disponível em:

<http://www.scielo.br/scielo.php?script=sci_arttext\&pid=S0080-62342011000200018>. Acesso em: 26 abr. 2014.

LYNN, P. Manual de habilidades de enfermagem de Taylor. Porto Alegre, Artmed, 2012.

MERRILL, M.D. First principles of instruction. Educational Technology Research and Development, v. 50, n. 3, p.:43-59, 2002.

ROY; R.B.; MCMAHON, G.T. Video-based cases disrupt deep critical thinking in problem-based learning. Medical Education,V. 46, p.: 426-435, 2012.

SALINAS, J.. Diseño, producción y evaluación de vídeos didácticos. Palma: Illes Balears, Universitat de lis Illes Balears, 1992.

SCHATKOSKI, A.M et al. hypertext, educational game, and simulation concerning oxygen therapy and itheir use by nursing students. an exploratory study. OBJN, Rio de Janeiro, v. 6, N.0, 2007. Disponível em:

<http://www.objnursing.uff.br/index.php/nursing/article/view/636/149> Acesso em: 15 jun 2014.

SILVA, A. P. S. S.; COGO, A.L. P.. Aprendizagem de punção venosa com objeto educacional digital no curso de graduação em enfermagem. Revista Gaúcha de Enfermagem, Porto Alegre, v. 28, n. 2, p.187-192, jun. 2007. Disponível em: <http://www.lume.ufrgs.br/bitstream/handle/10183/23577/000601139.pdf?sequence=1> . Acesso em: 24 abr. 2014. 
TORRES, M. M.; ANDRADE, D.; SANTOS, C. B.. Punção venosa periférica: avaliação de desempenho dos profissionais de enfermagem. Revista Latino-americana de Enfermagem, Ribeirão Preto, v. 13, n. 3, p.299-304, maio 2005. 